\title{
Simplified dressing change after loose combined cutting seton surgery for high anal fistula: a prospective, single center randomized controlled study
}

\author{
Jiaying Shan ${ }^{1}$, Lihua Zheng ${ }^{2}$, Yicheng Cheng ${ }^{2}$, Yuying Shi ${ }^{2}$, Congcong Zhi ${ }^{2}$, Yan Zhang ${ }^{1}$, Yaxuan Sun ${ }^{1}$, \\ Hongxin Guo ${ }^{1}$, Dun Liu ${ }^{1}$ \\ ${ }^{1}$ Graduate School, Beijing University of Traditional Chinese Medicine, Beijing, China; ${ }^{2}$ Department of Proctology, China-Japan Friendship Hospital, \\ Beijing, China \\ Contributions: (I) Conception and design: J Shan, L Zheng; (II) Administrative support: Y Cheng, Y Shi; (III) Provision of study materials or patients: \\ C Zhi, Y Zhang; (IV) Collection and assembly of data: Y Sun, H Guo, D Liu; (V) Data analysis and interpretation: J Shan, L Zheng; (VI) Manuscript \\ writing: All authors; (VII) Final approval of manuscript: All authors. \\ Correspondence to: Lihua Zheng. Proctology Department, China-Japan Friendship Hospital, Beijing 100029, China. Email: btv1262021@163.com.
}

\begin{abstract}
Background High anal fistula (HAF) is a refractory infectious disease. Surgery is the most effective way to treat HAF. Dressing change is an indispensable part of the rehabilitation process after surgery. The purpose of this study is to provide feasibility and evidence of safety for the implementation of a simplified dressing change after loose combined cutting seton (LCCS) surgery and to offer a better method for clinical treatment and postoperative rehabilitation of HAF.

Methods: In this single-blind randomized controlled trial, 76 patients diagnosed with HAF will be randomly divided into two groups: the simplified dressing change group $(n=38)$ or the traditional debridement and dressing change group $(n=38)$. Compared with traditional debridement and dressing change, simplified dressing change was conducted without mechanical debridement and disinfection. All patients were treated surgically with the LCCS and dressing change. Postoperative follow-up will be carried out on the $3 \mathrm{rd}, 7 \mathrm{th}, 14 \mathrm{th}, 21 \mathrm{st}$, and 180th day after the operation. The primary outcomes will be: complete healing rate of wound and fistula, long-term recurrence rate, poor wound healing rate, and complete wound healing time. The following secondary outcomes will be evaluated: postoperative pain using a visual analogue scale (VAS) score, wound secretions, edema, granulation shape, depth of wound, duration of each dressing change, and incidence of adverse events.

Discussion: Dressing change after HAF surgery is a necessary stage of recovery after anorectal surgery. Effective dressing change can reduce false healing and increase the cure rate. However, traditional dressing change takes a long time, and the patient endures severe pain. We have found that the dressing change process can be simplified in the clinic for patients treated with LCCS. In particular, simplification of the dressing change process may be related to the unobstructed drainage provided by the combination of LCCS and the separation of the dotted line. We will treat HAF using LCCS and compare the simplified dressing change method after the operation with traditional routine debridement and dressing change to demonstrate whether the simplified dressing change can be used in patients with HAF treated with LCCS.
\end{abstract}

Trial Registration: ChiCTR2100047312.

Keywords: Loose combined cutting seton (LCCS); simplified dressing change; high anal fistula (HAF); efficacy; recurrence

Submitted Aug 31, 2021. Accepted for publication Oct 20, 2021.

doi: $10.21037 /$ apm-21-2726

View this article at: https://dx.doi.org/10.21037/apm-21-2726 


\section{Introduction}

High anal fistula (HAF) can be defined as an anal fistula, which has a higher risk and cannot be safely treated using conventional fistula incision (1). In clinical practice, HAF is a refractory disease. Its lesions invade many muscles, and the pipes are packed and disjointed. The main pipe often passes through the deep part of the external sphincter and invades the puborectalis and levator ani muscle. HAFs can hardly heal by themselves and must be treated with surgery (2-4). The loose combined cutting seton (LCCS) method is based on the traditional Chinese medicine thread-hanging technique. After a long period of continuous improvement, it has developed into a new surgical method. Due to its high cure rate and low anal function loss rate, it has gradually become a common surgical method for the treatment of HAF in China. The LCCS method for HAF can reduce the damage to the sphincter while ensuring the function of the anal sphincter as much as possible. The solid thread mainly plays the role of cutting the sphincter. After the sphincter is partially suspended, the solid thread naturally becomes a virtual thread. The thread can drain the fistula and other necrotic tissues along the silk thread, play a good drainage effect, and is not prone to pseudo-healing (5-7).

Due to the deep location of HAF anal fistulas, postoperative wounds are large, the pain patients experience during a dressing change is severe, and patients resist anal contraction, which makes it more difficult to clean the dressing. The dressing of the wound after anal fistula surgery affects the success of the operation to a certain extent. According to wound infection or contamination, wounds can be divided into clean wounds, contaminated wounds, and infected wounds $(8,9)$. The wound of a HAF is an infected wound. Because intestinal fluids and feces pass through the wound, this type of wound is directly exposed to the bacterial infection and sinuses. There is continuous pus, exudate, and necrotic tissue drain from the inside to the outside. Dampness and fluid accumulation in the wound greatly increase the risk of infection. At the same time, the anus is easily squeezed by the large buttocks muscle tissue on both sides, has poor air permeability, and hence many bacteria can multiply. To avoid infection, care should be taken to ensure smooth drainage during and after surgery to avoid new infections caused by fluid accumulation in the dead space of the wound.

The basic principles of postoperative wound management for HFA are: (I) keep the perianal clean, (II) allow drainage to flow, and (III) prevent false healing. The purpose of dressing change is to eliminate the pus and necrotic tissue of the infected fistula, prevent the accumulation of blood and exudate in the intestinal cavity and anal canal leading to tissue damage and the re-formation of the local necrotic cavity, and avoid premature healing of the wound and the formation of false healing $(10,11)$. Traditional HAF dressing changes are based on the principle of aseptic technology. Doctors always work under strictly sterile conditions when washing and debriding the wound, packing drainage gauze and changing the dressing. Such dressing changes are performed daily (to be done 1-2 times a day) and the steps are cumbersome and time-consuming. In the clinic, we found that patients are very fearful when dressings are changed. We found that the secretions adhere to the wound during dressing change. The tearing and pulling of the wound when changing dressings destroy the new granulation tissue. Wiping the wound with a cotton ball can also cause severe pain due to the stimulation of peripheral nerves around the wound. Sphincter spasm pain is often caused when new drainage gauze is filled, which leads to severe pain and psychological fear of changing dressings (12-15). Due to the special anatomical location of HFAs, patients with anal fistulas take a long time to recover after surgery. Improper application of dressing changes will cause a significant waste of medical resources and greatly increase the cost of patient care. In addition, strict aseptic conditions are important for the operation during dressing changes. The demands on the environment, equipment, and personnel are high. Therefore, simplifying the dressing procedure without affecting the cure rate can not only reduce the patient's pain and save doctors' time but also shorten hospital stays and reduce in-hospital infection.

During LCCS the sphincter does not need to be completely cut off to retain the normal function of the anus, such that continuous, good and smooth drainage is achieved after the operation, which in turn not only reduces postoperative pain but also avoids poor drainage after the traditional hanging thread has fallen off (16-18). Studies have shown that using tap water to flush wounds does not increase the infection rate compared to normal saline. Flushing can also help patients to freely adjust water pressure and temperature according to their pain conditions and reduce the pain of patients' wounds during washing (19-21). Therefore, the treatment of HAF patients through the LCCS can fully guarantee drainage and reduce the incidence of false healing. We can greatly simplify the dressing procedure and only perform necessary wound cleaning and external packing dressings to absorb 
excessive secretions. Therefore, we will undertake a singlecenter randomized controlled trial to compare the clinical efficacy of simple dressing change with that of traditional debridement and dressing change and assess the incidence of postoperative complications, so as to provide clinicians with more data on which to base clinical decisions.

\section{Study objectives}

In this study, we will compare the clinical efficacy of a simplified dressing change method and the traditional debridement dressing method in the treatment of postoperative wounds of HFAs. We hope that the results from this trial will provide the basis for the clinical implementation of the simplified dressing change.

\section{Study design}

The study is a prospective, randomized controlled, singleblind, and single-center non-inferiority clinical trial. We present the following article in accordance with the SPIRIT reporting checklist (available at https://dx.doi.org/10.21037/ apm-21-2726).

\section{Methods}

\section{Protocol writing}

The writing of this article is based on the recommendations of the relevant clinical trial protocol and related documents published in the SPIRIT 2013 statement in the "Journal of Evidence-Based Medicine in Traditional Chinese Medicine" (22). All procedures performed in this study involving human participants will be in accordance with the Declaration of Helsinki (as revised in 2013).

\section{Study settings}

This study will enroll patients who have been diagnosed with HFAs in the Anorectal Clinic of the China-Japan Friendship Hospital (Beijing, China) and have been planned to be admitted to the hospital for surgical treatment.

\section{Enrollment and eligibility criteria}

All patients diagnosed with HFAs can participate in this study. The Western medicine diagnostic criteria are in accordance with the "Guidelines for the Clinical Diagnosis and Treatment of Perianal Abscesses, Anal Fistulas, and Rectovaginal Fistulas" compiled by the American Association of Colorectal Surgeons in 2016 (23). The traditional Chinese diagnostic criteria are in accordance with the 2012 Chinese Society of Chinese Medicine's "Guidelines for the Diagnosis and Treatment of Common Diseases in the Anorectal Department of Traditional Chinese Medicine" (24).

\section{Inclusion criteria}

Participants must meet all of the following criteria to be included in this study: meet the diagnostic criteria for HAF; have received LCCS treatment; have normal anal function; are aged 18-65 years; show good compliance; fully understand the purpose, methods and significance of this study; volunteer to participate in this study; and sign an informed consent form.

\section{Exclusion criteria}

Potential subjects who meet any one of the following criteria will be excluded from this study: patients with nonHAF; patients not treated with LCCS; suffering from active inflammatory bowel disease (ulcerative colitis and Crohn's disease); and pregnant or lactating women.

\section{Intervention procedures}

The research process is divided into three stages.

The first stage is the screening period. The main task will be to evaluate cases according to the inclusion and exclusion criteria and to sign the informed consent form before inclusion. Before being selected for the study, the doctor will ask and record the patient's medical history and perform screening examinations such as digital rectal examination and intrarectal ultrasound.

The second stage is the treatment period, which mainly includes: (I) preoperative preparations: The two groups will undergo the same preoperative preparations, that is anorectal cavity color Doppler ultrasound and other examinations before the operation; the diagnosis will be confirmed; it will also be confirmed that the patient has no contraindications for surgery; the patient will fast for 8 hours prior to surgery, empty the bowels normally, and will be given an enema if necessary. (II) Surgical operation: Both groups of patients will be treated with LCCS. The patient will assume a lateral position, and after intravenous 
anesthesia, the operation area will be disinfected and draped. Once the anal canal will have become loose, the anal canal will be disinfected and an anorectal exploration will be performed to determine the area and location of the anal fistula, and whether there are branches and dead spaces. During the operation, a radial incision will be made from the inner orifice outward from the dentinal line, about 3 to $4 \mathrm{~cm}$ in length, and the incision position will generally be on the same side as the outer orifice to fully drain the infection foci at the inner orifice. The inner orifice will be cut, extending $0.5-1.0 \mathrm{~cm}$ upwards, and extending downwards to the outside of the anal margin. Curved hemostatic forceps will be used to probe the upper end of the fistula from this incision until the top of the fistula. Fingers will be used to reach into the intestinal cavity for guidance, and the tip of the forceps will be employed to penetrate the stoma of the intestinal wall. The finger will be withdrawn, four 10-gauge silk threads will be used, with one end tied to the fingertip, and placed into the intestinal cavity. The hemostatic forceps will be opened to clamp the thread, the silk thread will be drawn from the intestinal cavity through the fistula, and the two ends will be gathered and secured with a knot. Routine cleaning will be carried out and the dressing will be changed once a day after the operation. The Vaseline gauze will be drained, and the gauze will be applied and secured. About 7 days after the operation, the hanging silk thread will be loose. At this time, the thread will be kept hanging there without tightening. After the granulation tissue of the fistula will have been filled, the thread will be removed on the 20th day. (III) Postoperative medication: Both groups of patients will receive routine intravenous infusions of broad-spectrum and anti-anaerobic antibiotics for 3 days after surgery. (IV) The dressing on wounds will be changed after surgery: for those who defecate within 24 hours after the operation, the dressing is changed immediately after defecation. For those who have a bowel movement after more than $24 \mathrm{~h}$, the dressing shall be changed $24 \mathrm{~h}$ after the operation. After defecation, both groups will use Chinese medicine preparations to fumigate and wash for 10 minutes. The prescription of Chinese medicine preparations for fumigation and washing will be: $15 \mathrm{~g}$ of Chinese gallnut, $30 \mathrm{~g}$ of dandelion, $15 \mathrm{~g}$ of arborvitae, $30 \mathrm{~g}$ of Sophora flavescens, $15 \mathrm{~g}$ of Glauber's salt, $15 \mathrm{~g}$ of Atractylodes, $15 \mathrm{~g}$ of sanyu, $15 \mathrm{~g}$ of fangfeng, $30 \mathrm{~g}$ of Phellodendron 30, $15 \mathrm{~g}$ of red peony, $15 \mathrm{~g}$ honeysuckle, $10 \mathrm{~g}$ raw licorice, one dose a day each. Usage: These will be wrapped with gauze, put it in a basin, and brewed with 2,000 $\mathrm{mL}$ hot water. When the water temperature is about $40{ }^{\circ} \mathrm{C}$, the anus and wound will be washed for 10 minutes, twice a day.

After fumigating and washing in the sitz bath, the dressing will be changed. The dressing change method will be adapted based on the plan that will be made at the time of enrollment. A. Intervention group: The simplified dressing change method will be used. Preparation before operation: The operator will wash his/her hands and will wear a mask and a hat. He/she will inform the patient about the purpose of the dressing change and obtain the patient's cooperation. A disposable sterile dressing change box (containing 2 curved trays, 1 pad towel, 2 tweezers, gauze, and cotton balls) will be set aside in addition to medical tape. Operation steps: (I) the old dressing will be removed from the wound: The patient will be instructed to assume a lateral position (to fully expose the perianal drainage wound), the disposable dressing pack will be opened, and the items will be set aside. First, the outer dressing will be removed by hand, and then a pair of tweezers will be used to remove the inner dressing and the stuffed cotton ball. If it adheres to the wound surface, care will be taken that it does not peel off too vigorously. It will be soaked with a normal saline cotton ball and removed to avoid injury and bleeding. (II) The wound will be washed: After instructing the patient to sit in the bath, the contralateral buttocks muscle will be moved firmly with one hand to fully expose the wound. The shower will be held with the other hand, or the shower will be applied by a family member using tap water (set temperature is $36-37^{\circ} \mathrm{C}$ ) within $5 \mathrm{~cm}$ from the wound; and the wound and the skin within $5 \mathrm{~cm}$ from the wound will be washed under pressure, with the order being from top to bottom, from the inside to the outside, the wound surface will be washed, the solid line will be pulled gently to locate the wound cavity, and the dotted line will be rotated to wash the necrotic tissue attached to the line until the pus on the wound surface has been completely washed out. (III) Change dressing: Sterile cotton balls or sterile gauze will be placed in each wound cavity, whereby the outer layer will not need to be covered with sterile gauze. Patients and family members will pay attention to the dry cotton balls placed in the wound cavity and replace it immediately once it is soaked with secretions and pus. Cotton balls and gauze will be used to fill local wound defects in the perianal area to prevent the wound from healing earlier than the wound cavity, leading to pseudo-healing and forming a new sinus tract. A hanging thread will be able to provide adequate drainage, allow excessive exudate and pus to be fully discharged, provide a relatively moist rather than humid environment for wound healing, and reduce edema of the 
tissue around the wound (25).

B. Control group: traditional debridement and dressing change will be used. Preparation before operation: the dressing operator washes will wash his/her hands and will wear a mask and a hat. The patient will be informed about the purpose of the dressing change and the patient's cooperation will be obtained. Preparation before operation: a disposable sterile dressing change box (containing two curved trays, one pad towel, two tweezers, gauze and cotton balls), one bottle of iodophor, several petroleum jelly sticks, one bottle of saline, one pair of curved forceps, one pair of curved scissors, one syringe $(50 \mathrm{~mL})$, and medical tape. The operation will be conducted as follows: (I) the previous dressing will be removed from the wound, the patient will be instructed to assume a lateral position (to fully expose the perianal drainage wound), the disposable dressing pack will be opened, and the items will be set aside. First, the outer dressing will be removed by hand, and then a pair of tweezers will be used to remove the inner dressing. If it adheres to the wound surface, care will be taken to not remove it too vigorously. It will be soaked with a cotton ball of saline and removed to avoid injury and bleeding. Tweezers will be used to slowly pull out the old petroleum jelly gauze along the long axis of the wound. (II) Mechanical debridement: Because the infected wound has more secretions and the wound is deep, the patient is asked to forcefully break the contralateral buttocks muscle with one hand to fully expose the wound. A syringe will be used to draw physiological saline, $5 \mathrm{~cm}$ away from the wound, and the wound and the surrounding $5 \mathrm{~cm}$ area will be flushed under pressure (26), with the order being from top to bottom, from the inside to the outside, gently pulling the solid line to locate the wound cavity and rotating the dotted line to wash the attached necrotic tissue until the pus on the wound surface has been thoroughly rinsed, and a cotton ball will be used to wipe the wound with curved forceps to remove slough, tissue fragments, foreign bodies on the wound, and reduce the growth of bacteria on the wound until the wound is rosy, and there is a trace of blood exudation. (III) Wound disinfection: Sterile gloves will be worn, the tweezers will be held in the left hand to keep the wound aseptic, and the tweezers will be held in the right hand to touch the wound. An iodophor cotton ball will be used to disinfect the skin and wound area within $5 \mathrm{~cm}$ of the wound from the outside to the inside three consecutive times. The wound and the surrounding $5 \mathrm{~cm}$ skin will be wiped with a cotton ball of isotonic saline. The sterile curved forceps will be held, and the wound will be blotted with gauze. (IV) Packing the drainage strip: When plugging the drainage strip, this will start from the bottom of the wound, and curved forceps will be used to pack up along the long axis of the perianal radial wound until it will have reached the highest point of the fistula. It should not be too tight or too loose. If it is too tight, this will affect the drainage of pus. If it is too loose, it will easily fall off the wound. The end of the oil gauze will be left outside the wound, such that it can be taken out and drained smoothly during the next dressing change. (V) Changing the dressing: Eight to 10 layers of sterile gauze will cover the wound and will be fixed with tape. The direction of the fixation will be perpendicular to the long axis of the body. If there are more secretions and pus, a layer of cotton pad will be covered over the sterile gauze.

Both groups of patients will have their dressing changed twice a day, once in the morning and once in the evening. (Dressings should be changed within half an hour after defecation every day. If there is no bowel movement that day, the first dressing change will be performed at 9-10 am and the second dressing change at 21-22 pm).

The third period is the follow-up period. During the hospitalization period, the patients and their families will be trained in dressing change (Appendix 1: training guide for patient changing dressing). Follow-up plan: The follow-up time is half a year, including six medical visits. Telephone follow-up or face-to-face follow-up will be conducted on the first consultation day, and the $3 \mathrm{rd}$, 7 th, 14th, 21st, and 180th day after surgery. The main contents include: patient's current symptoms, anorectal ultrasound, rectal pressure measurement, and anal function score. The Wexner Incontinence Score will be measured.

\section{Outcome measures and data collection}

The main outcomes will be: postoperative wound and fistula complete healing rate, long-term recurrence rate, poor wound healing rate, and complete wound healing time. Secondary outcomes to be evaluated will be: postoperative pain visual analogue score (VAS) score, wound secretion, edema, granulation morphology, wound depth, the duration of each dressing change, and the incidence of adverse events.

\section{Primary outcomes}

Main indicator of curative effect: complete cure rate of postoperative wounds and fistulas. 


\section{Secondary outcomes}

These will include secondary efficacy evaluation indicators and safety evaluation indicators. The secondary efficacy evaluation indicators will include: (I) six months of recurrence, (II) poor wound healing rate, and (III) anorectal ultrasound. Safety evaluation indicators will include: (I) VAS score for postoperative pain, (II) measurement of anal and rectal pressure before and after treatment, (III) assessment of anal function before and after treatment: Wexner anal function assessment, and (IV) adverse events.

\section{Sample size}

This study will be a non-inferiority clinical randomized controlled trial. The cure rate will be the main outcome indicator, and $\mathrm{N}$ will be the sample size. The ratio of the test group and the control group is 1:1. According to previous literature reports and clinical application summaries, the cure rate will be set to be $95 \%$. The statistical tests will use P2 $=0.95$ for the control group, $\alpha=0.025$ (one-sided), and the power will be set at $80 \%$. The non-inferiority margin will be $d=0.15$. Using PASS15 software, the sample size of each group will be 34 . Assuming that the loss of follow-up rate of the research subjects is $10 \%$, the sample size of each group will be $\mathrm{N}=34 / 0.9=38$.

\section{Recruitment}

Recruitment for this trial will be carried out through the internet and public release of the subject recruitment information, and recruitment materials will be placed in outpatient clinics. After registration, subjects will be enrolled after having passed through the regular medical treatment process and screening using the admission and discharge criteria.

\section{Method of allocation of interventions}

\section{Randomization and blinding}

Before enrollment, we will tell patients that during the treatment process, the surgeon will use the LCCS to perform the operation. The postoperative dressing change method will be the traditional method or the simplified method. The dressing change method will be specifically determined by the doctor. To minimize the bias of the trial, after the clinical follow-up has been completed, the data will be sent to a third party for statistical analysis. The third party will not know which data belongs to which group before the results are generated. Finally, the physician and the third party performing the statistical analysis will jointly announce the results relating to each group.

Randomization method: random allocation will be undertaken using cards according to a random number table, which will have the random number of the serial number and the group allocation written on them. The random distribution card will be placed into a special opaque kraft paper bag with a serial number, and it will be sealed, and the envelopes will be opened in sequence after the patients will have been admitted to the hospital according to the admission criteria. Group A is the experimental group, where the simplified dressing change method will be used. Group B will be the control group, where traditional debridement and dressing change will be used.

\section{Statistical analysis}

The statistical software package SPSS 20.0 will be used for statistical analysis. All statistical tests will be performed using two-sided tests, and a $\mathrm{P}$ value of $<0.05$ will be considered statistically significant.

The mean, standard deviation or median, and interquartile range of blood pressure, heart rate, body temperature, measured value of anorectal pressure, wound and fistula, and finally healing time will be described. The number of cases and the percentage of classification indicators such as gender, wound pain degree score, anal function evaluation score, and curative effect evaluation results will be described. The Kaplan-Meier method will be used to estimate the healing rate at each time point after surgery, and the log rank test method will be used to compare the healing times between the two groups.

The mean \pm standard deviation will be used to describe the resting pressure of the anorectal anal canal, the length of the anal canal high pressure area, and the maximum systolic pressure of the anal canal before treatment and after healing in the experimental group and the control group, respectively. The above indicators before treatment will be used as covariates, and analysis of covariance will be used to compare whether the above indicators are statistically different between the test group and the control group after surgery.

To compare quantitative data between two groups, for example, when comparing the final healing time of wounds and fistulas between the test group and the control group, normality and homogeneity of variance will be considered. 
If normality and uniform variance are satisfied, a $t$-test is used, if not, a t'-test is used. A Wilcoxon rank sum test will be used for non-normally distributed data. To compare categorical data between the two groups, such as the results of anal function curative effect evaluation, the $\chi^{2}$ test will be used, and for grade data the rank sum test. The paired signed-rank test will be used to compare the changes of incontinence scores before and after treatment in each group, and the one-way ordered $\chi^{2}$ test will be used to compare the groups.

\section{Record of adverse events}

We will record adverse events during clinical trials. Serious adverse events will be defined as: events that require hospitalization, prolonged hospitalization, disability, ability to work compromised, life-threatening conditions or death, and congenital malformations occur during the trial. Clinical adverse events may occur during the treatment of subjects. Once adverse events (including important adverse events) occur, the time of occurrence, clinical manifestations, treatment process and duration, outcome, and related events will be recorded in detail on the case report form. If there is an abnormal laboratory test, the patient must be followed up until the test result will have returned to normal or to the level before the treatment, or it is determined that it has nothing to do with the treatment. Serious adverse events will be recorded on the serious adverse event form and reported to the ethics committee within 24 hours.

\section{Ethics committee review}

This protocol, written informed consent, and materials directly related to the subjects will be submitted to the ethics committee, and the research will be formally carried out after obtaining the written approval of the ethics committee. The investigator will submit a research report to the ethics committee at least annually (if applicable). When the research is suspended and/or completed, the researcher will notify the ethics committee in writing; the researcher will report to the ethics committee all changes in the research (such as the revision of the protocol and/or the number of informed consents). These changes may not be implemented until approved by the committee, unless they will be made to eliminate obvious and direct risks to the subject. In such cases, the ethics committee will be notified.

\section{Informed consent}

\section{Procedure for obtaining informed consent}

The researcher will provide the subjects or their legal representatives with an easy-to-understand informed consent form approved by the ethics committee and give the subjects or their legal representatives sufficient time to consider this research. Subjects will not be allowed to be allocated to their group before their signed written informed consent has been obtained. During the participant's participation, all updated informed consent forms and written information will be provided to the participants. The informed consent form will be kept as an important document for clinical trials for future reference.

\section{Confidentiality measures}

The results of this research project may be published in medical journals, but we will keep the patient's information confidential in accordance with the legal requirements, and the patient's personal information will not be disclosed unless required by relevant laws. When necessary, government and hospital ethics committees will be able to access patients' data in accordance with regulations.

\section{Ethics and dissemination}

This study was approved by the Institution Review Board of China-Japan Friendship Hospital (approval number: 2020-89-K53). The results of this study will be submitted to international scientific peer-reviewed journals or conferences in surgery, anorectal surgery, or anorectal diseases.

\section{Discussion}

How to protect the integrity of the sphincter and maintain the normal function of the anus is the biggest difficulty in the surgical treatment of HAFs. Doctors have different treatment approaches for HAFs. Some foreign doctors and scholars advocate surgical procedures that preserve the sphincter, such as rectal flap operation, intersphincteric fistula ligation, anal fistula clogging, loose threading, and fibrin glue occlusion (27-31). The problem is that the fistula between the sphincter muscles may become a new focus of infection. Domestic anorectal doctors advocate that the sphincter be preserved as much as possible, while the fistula should be removed. Therefore, the thread-hanging operation has emerged. As a traditional surgical treatment in China, written records existed of the clinically application 
of thread-hanging therapy as early as the Yuan Dynasty. After the continuous improvement and development of this technique by doctors during past centuries, there are currently two types of mainstream clinical thread-hanging methods: real thread-hanging and virtual thread-hanging. Through tightening of the thread and using its contraction, the elastic force slowly squeezes the sphincter. The disadvantage is that the patient feels as if a foreign body hangs on the thread, the pain is heavier, and the healing time is longer. The virtual thread draws only the drainage effect of the silk thread and does not cut the sphincter. The patient's pain is significantly reduced compared with the cutting thread method, and the anus control function is better after the operation. Due to the incomplete treatment of the infection foci, as the follow-up time increases, the postoperative recurrence rate gradually increases, which leads to an increased risk of the patient's second surgery (32).

The LCCS method adopts the silk thread hanging thread, which combines the advantages of real thread hanging and virtual thread hanging. During the operation, the real thread hanging usually uses the silk thread without elastic effect. Silk thread reduces the suffering of patients. After the operation, the necrotic tissue falls off, the real hanging becomes empty, and the drainage is continued during the recovery process. The long thread tail is stuffed in the incision and drainage under the anal straight ring, which can also prevent false healing. This technique has been used in clinical practice for many years, which has been proven effective and safe. The pain score, cure rate, and recurrence rate of the patients is better than those of traditional thread-hanging surgery $(6,7,18)$. In the clinic, we found that many patients who were discharged early after the operation due to family or work reasons did not have time to return to the hospital frequently for dressing changes. In these cases, the dressing was changed at home. The wounds of these patients also healed well, and no pseudohealing was found. These observations fully demonstrated good drainage and prevention of false healing of the LCCS method. At the same time, studies have shown that repeated or excessive use of disinfectants can affect normal human cells and adversely affect normal tissue repair (33). Therefore, we put forward the idea of simplifying dressing change and formulated a patient dressing change training plan to reduce the pain of patients, reduce the length of hospital stays, and improve the efficiency of specialist medical treatment. Since we abandon the traditional debridement and disinfection, more attention on the wounds should be paid in nursing care after LCCS to make sure the wounds heal.

The purpose of our single-center randomized controlled trial is to further verify the effectiveness and safety of simplified dressing change after LCCS treatment to support its clinical application.

\section{Acknowledgments}

Funding: This study was funded by the Wu Jieping Medical Foundation (Project number: 320.6750.2020-8-34).

\section{Footnote}

Reporting Checklist: The authors have completed the SPIRIT reporting checklist. Available at https://dx.doi. org/10.21037/apm-21-2726

Conflicts of Interest: All authors have completed the ICMJE uniform disclosure form (available at https://dx.doi. org/10.21037/apm-21-2726). All authors reported they received support from $\mathrm{Wu}$ Jieping Medical Foundation (Project number: 320.6750.2020-8-34). The authors have no other conflicts of interest to declare.

Ethical Statement: The authors are accountable for all aspects of the work in ensuring that questions related to the accuracy or integrity of any part of the work are appropriately investigated and resolved. This study was approved by the Institution Review Board of China-Japan Friendship Hospital (approval number: 2020-89-K53). All procedures performed in this study involving human participants will be in accordance with the Declaration of Helsinki (as revised in 2013). This protocol, written informed consent, and materials directly related to the subjects will be submitted to the ethics committee, and the research will be formally carried out after obtaining the written approval of the ethics committee.

Open Access Statement: This is an Open Access article distributed in accordance with the Creative Commons Attribution-NonCommercial-NoDerivs 4.0 International License (CC BY-NC-ND 4.0), which permits the noncommercial replication and distribution of the article with the strict proviso that no changes or edits are made and the original work is properly cited (including links to both the formal publication through the relevant DOI and the license). See: https://creativecommons.org/licenses/by-ncnd $/ 4.0 /$. 


\section{References}

1. An AM. Anorectology. Beijing: People's Medical Publishing House, 1998:121.

2. Chen XL, Feng LQ, Jiang GD, et al. Expert consensus on the diagnosis and treatment of anal fistula (2020 edition). J Prac Clin Med 2020;24:1-7.

3. Zong Z, Chen S. Progress in the treatment of high complex anal fistulas. Lingnan Moder Clin Surg 2012;12:155-8.

4. D'Hoore A, Penninckx F. The pathology of complex fistula in ano. Acta Chir Belg 2000;100:111-4.

5. Wu XX. Analysis on the treatment of anal fistula with thread-drawing therapy in traditional Chinese medicine. Jiangxi Traditional Chinese Medicine 2019,50:15-7.

6. Shi YY, Zheng LH, Zhang W, et al. Discussion on the results of anorectal 3D pressure measurement and anal incontinence WEXNER score after high anal fistula combined with thread-drawing operation. Chin J Clinicians 2019,47:1076-8.

7. Zheng LH. Using isobaric drainage theory to treat high anal fistula by combining virtual and actual with thread-hanging method. Anorectal branch of Clinical Education Research Association of Chinese Medicine Higher Education Association and Anorectal Professional Committee of Hubei Chinese Medicine Association 2016 Academic Annual Conference Proceedings 2016:139-43.

8. Liu Q, Cao ZJ. Research progress in dressing change for wounds. J Trau Surg 2007;9:183-4.

9. Diegelmann RF, Evans MC. Wound healing: an overview of acute, fibrotic and delayed healing. Front Biosci 2004;9:283-9.

10. Li YN. Chinese Anorectology. Chongqing: Science and Technology Press Chongqing Branch, 1990:1.

11. Wu ZP, Ma D, Deng L, et al. Discussion on dressing change after anorectal disease. Chin J Anorectal Dis 2012;32:70-1.

12. Kirshen C, Woo K, Ayello EA, et al. Debridement: a vital component of wound bed preparation. Adv Skin Wound Care 2006;19:506-17; quiz 517-9.

13. Zhang YY, Chen XY. Research progress in pain management during wound debridement. Chin J Moder Nurs 2012;18:615-7.

14. Bowler PG, Duerden BI, Armstrong DG. Wound microbiology and associated approaches to wound management. Clin Microbiol Rev 2001;14:244-69.

15. Spichler A, Hurwitz BL, Armstrong DG, et al. Microbiology of diabetic foot infections: from Louis
Pasteur to 'crime scene investigation'. BMC Med 2015;13:2.

16. Liu HQ. The effect of Kangfuxin liquid on wound healing after operation for low-position simple anal fistula. Herald Chin Med 2011;17:58-9.

17. Zheng L, Shi Y, Zhi C, et al. Loose combined cutting seton for patients with high intersphincteric fistula: a retrospective study. Ann Transl Med 2020;8:1236.

18. Shi YY. Clinical observation and experimental study on the treatment of high intersphincter anal fistula with combination of deficiency and excess and thread-drawing. Beijing University of Chinese Medicine, 2020.

19. Zhao YM. A retrospective cohort study of anal function after anal fistula thread-drawing operation. Fujian University of Traditional Chinese Medicine, 2020.

20. Fernandez RS, Griffiths R, Ussia C. Water for wound cleansing. Int J Evid Based Healthc 2007;5:305-23.

21. Moscati RM, Mayrose J, Reardon RF, et al. A multicenter comparison of tap water versus sterile saline for wound irrigation. Acad Emerg Med 2007;14:404-9.

22. Chan AW, Tetzlaff JM, Altman DG, et al. SPIRIT 2013 statement: defining standard protocol items for clinical trials. Ann Intern Med 2013;158:200-7.

23. Obilor HN, Adejumo PO, Ilesanmi RE. Assessment of patients' wound-related pain experiences in University College Hospital, Ibadan, Nigeria. Int Wound J 2016;13:697-704.

24. Zhao XY, Liu MY, Han XJ, et al. Clinical application evaluation of Guidelines for Diagnosis and Treatment of Common Diseases of Coloproctology in Traditional Chinese Medicine. Zhongguo Zhong Yao Za Zhi 2017;42:3252-6.

25. Eaglstein WH. Moist wound healing with occlusive dressings: a clinical focus. Dermatol Surg 2001;27:175-81.

26. Longmire AW, Broom LA, Burch J. Wound infection following high-pressure syringe and needle irrigation. Am J Emerg Med 1987;5:179-81.

27. Rizzo JA, Naig AL, Johnson EK. Anorectal abscess and fistula-in-ano: evidence-based management. Surg Clin North Am 2010;90:45-68, Table of Contents.

28. Cavanaugh M, Hyman N, Osler T. Fecal incontinence severity index after fistulotomy: a predictor of quality of life. Dis Colon Rectum 2002;45:349-53.

29. van Koperen PJ, Wind J, Bemelman WA, et al. Fibrin glue and transanal rectal advancement flap for high transsphincteric perianal fistulas; is there any advantage? Int J Colorectal Dis 2008;23:697-701.

30. Loungnarath R, Dietz DW, Mutch MG, et al. Fibrin glue 
treatment of complex anal fistulas has low success rate. Dis Colon Rectum 2004;47:432-6.

31. Pigot F. Treatment of anal fistula and abscess. J Visc Surg 2015;152:S23-9.

32. Wang L, Gu YF. Clinical research progress of sphincterpreserving surgery in the treatment of complicated anus.

Cite this article as: Shan J, Zheng L, Cheng Y, Shi Y, Zhi C, Zhang Y, Sun Y, Guo H, Liu D. Simplified dressing change after loose combined cutting seton surgery for high anal fistula: a prospective, single center randomized controlled study. Ann Palliat Med 2021;10(10):11156-11165. doi: 10.21037/apm-212726
Chongqing Med 2016;45:1851-3.

33. Atiyeh BS, Dibo SA, Hayek SN. Wound cleansing, topical antiseptics and wound healing. Int Wound J 2009;6:420-30.

(English Language Editor: B. Meiser) 


\section{Appendix 1: training guide for patient changing dressing}

\section{What is bigh anal fistula?}

Anal fistula refers to an abnormal duct formed by the rectum or anal canal communicating with the perianal skin due to pathological reasons. It is also called anorectal fistula. Anal fistula is one of the most common anorectal diseases in China. The total incidence of anorectal diseases in China is $59.1 \%(33,837 / 57,297)$. The incidence of anal fistula is second only to hemorrhoids. The incidence of anal fistula in China accounts for $1.67 \%$ to $3.60 \%$ of the disease, $8.00 \%$ to $25.00 \%$ abroad, the majority of young and middle-aged people aged 20-40 years are affected, and the prevalence of males is higher than that of females. Among them, HAF is a type of anal fistula, which usually refers to a type of anal fistula in which the fistula of the anal fistula passes through the levator ani muscle. Most HAFs develop from perianal abscesses. If patients do not seek medical treatment in time, perianal abscesses will gradually form fistulas in the acute phase, which will not heal and recur. The main clinical symptoms are repeated perianal discharge or fecal discharge, pain, itching, rectal tenderness, and a sinking sensation. When the patient touches the anus and surrounding tissues, the perianal induration can be palpable, including the fistula leading to the anus. High anal fistulas often invade the puborectalis and levator ani muscles through the deep part of the external sphincter. High anal fistulas are almost impossible to heal by themselves and must be treated with surgery.

\section{What is the LCCS method?}

At present, the mainstream methods of threading HAF in clinical practice are divided into two types: real threading and virtual threading. Rubber bands are usually used for a hanging thread, which is used for cutting, and the thread will be tightened continuously after the operation, and thus the sphincter muscle will be slowly severed using the elastic force ensuing from contraction. The disadvantage is that the patient feels as though a foreign body is hanging on the thread, the pain is heavier, and the healing time is longer. The virtual thread only exerts the drainage effect of the silk thread and does not cut the sphincter. The patient's pain is significantly reduced compared with the cutting thread method, and the anus control function is better after the operation. Due to the incomplete treatment of the infection foci, as the follow-up time increases, the postoperative recurrence rate gradually increases, which leads to an increased risk of the patient's second surgery.

The LCCS method combines the advantages of real thread and virtual thread. During the operation, the actual thread is threaded, and the silk thread has no elastic effect. Under anesthesia, the thread is tightened once to reduce the patient's pain. The necrotic tissue falls off after the operation, the silk thread becomes loose around 7 days after the operation, and thus the actual hanging becomes virtual hanging, and the drainage continues during the recovery process. At this time, the virtual hanging thread continues to be drained, and after the granulation tissue of the fistula has been filled, it is removed on the 20th day.

\section{When will the dressing be changed?}

The patient will begin to change the dressing twice a day 24 hours after the operation, once in the morning and once in the evening. (The dressing should be changed within half an hour after defecation every day. If there is no bowel movement that day, the first dressing change will be performed at 9-10 am and the second dressing change at 21-22 pm).

\section{How to identify the type of thread-hanging in LCCS therapy?}

There are three types of thread hanging after operation. The first type is the thread, which extends into the anus. This thread is hung on the sphincter to cut off part of the sphincter. When washing, this kind of thread only needs to be gently shaken to prevent the threads from being entangled. The second type of thread is the drainage thread. This thread generally loops around the anus and runs through the two incisions to provide smooth drainage. It is similar to a circular ring. It is necessary to gently rotate the thread during cleaning to help secretions and necrotic substances to discharge. The third type of thread is the intraoperative hemostatic thread. The ligation thread of this type of thread is short, and it is not easy to locate it. When washing and changing the dressing, it does not need to be processed. Vigorous wiping and tearing should be avoided to prevent the thread from falling off and bleeding.

\section{How to wash the wound?}

The flushing operation can be performed by the family or the patient him- or herself. The patient adjusts the water temperature and flushing intensity of the flusher. The water temperature is generally slightly higher than the 
body temperature of 36 to $37^{\circ} \mathrm{C}$, such that there will be no irritation caused by the water temperature being too low or to high, and the flushing intensity should not cause any pain during washing. When washing, the patient takes a squatting or sitting position to fully expose the wound and wash by him- or herself or by a family member holding the showerhead. The washing height is about $5 \mathrm{~cm}$ away from the wound. The washing range is the wound and the skin around it, and the wounds are flushed under pressure. The wound should be washed with pressure, and the sequence is as follows: from top to bottom and from the inside to the outside. During the cleaning process, you can gently shake the solid line to locate the wound cavity and rotate the dashed line to wash the necrotic tissue attached to the line until the pus on the wound surface is completely washed out.

\section{What are the specific steps to simplify the dressing change method?}

(I) Remove the old dressing from the wound: The patient assumes a lateral position (to fully expose the perianal drainage wound), opens the disposable dressing pack, and puts the items in place. First, the outer dressing is removed manually, and then a pair of tweezers is used to remove the inner dressing and the stuffed cotton ball. If it adheres to the wound surface, do not peel it off too hard. Soak it with cotton ball with normal saline and remove it to avoid injury and bleeding. (II) Wash the wound: After instructing the patient to sit in the bath, firmly move the contralateral buttocks muscle with one hand to fully expose the wound. Hold the shower head with the other hand or ask a family member to use the shower head with tap water (set temperature is $36-37^{\circ} \mathrm{C}$ ), $5 \mathrm{~cm}$ away from the wound, and wash the wound and the skin within $5 \mathrm{~cm}$ from the wound under pressure, in the order of from top to bottom and from the inside to the outside. Wash the wound surface with pressure, gently pull the solid line to locate the wound cavity and rotate the dotted line to wash the necrotic tissue attached to the line until the pus on the wound surface is completely washed out. (III) Change dressing: Place sterile cotton balls or sterile gauze in each wound cavity, whereby the outer layer does not need to be covered with sterile gauze. Instruct patients and family members to pay attention to the dry cotton balls placed in the wound cavity, and replace these balls once they are soaked with secretions. Because cotton balls and gauze fill the local wound defect around the anal area, prevent the wound from healing earlier than the wound cavity, which will lead to pseudohealing and formation of a new sinus tract. A suspended thread can provide adequate drainage, allow excessive exudate and pus to be fully discharged, provide a relatively moist rather than humid environment for wound healing, and reduce edema of the tissue around the wound. 